\title{
PAT protein mRNA expression in primary rat hepatocytes: Effects of exposure to fatty acids
}

\author{
ELENA GRASSELLI ${ }^{1}$, ADRIANA VOCI $^{1}$, CARLO PESCE $^{2}$, LAURA CANESI $^{1,3}$, \\ EMILIA FUGASSA ${ }^{1}$, GABRIELLA GALLO $^{1}$ and LAURA VERGANI ${ }^{1,3}$ \\ ${ }^{1}$ Department of Biology; ${ }^{2}$ DISTBIMO, University of Genova, Genova; ${ }^{3}$ INBB, Rome, Italy
}

Received September 8, 2009; Accepted October 26, 2009

DOI: 10.3892/ijmm_00000370

\begin{abstract}
Excess energy is stored as neutral lipids in lipid droplets (LDs) whose surface is coated by PAT proteins, each playing a distinct cellular function. The adipocyte differentiation-related protein (ADRP) and tail-interacting protein (TIP47) are expressed almost ubiquitously, whereas the oxidative tissue-enriched PAT protein (OXPAT) is expressed in specific tissues, such as the liver. In rat liver, only ADRP expression has been documented. This study was aimed at identifying OXPAT and TIP47 transcripts in rat hepatocytes, and investigating how their expression is modulated by excess lipids, using fat-enriched hepatocytes to mimic different degrees of steatosis. Primary rat hepatocytes were exposed to fatty acids (FFAs) for 12, 24 and $36 \mathrm{~h}$. Lipid accumulation was estimated by spectrophotometric quantification of triacylglycerol. Expression of PAT proteins as well as of PPAR $\gamma$ was evaluated by real-time RT-PCR. Hepatocytes exposed to FFAs showed progressive lipid accumulation. The increase in lipid content was associated with the induction of PAT protein expression. At $12 \mathrm{~h}$, OXPAT and TIP47 mRNA expression was up-regulated. At longer times, the level of OXPAT transcripts remained high, whereas that of TIP47 slowly declined. Conversely, ADRP expression showed a time-dependent increase with exposure to FFAs. This study demonstrates, for the first time, the presence of OXPAT and TIP47 transcripts in rat hepatocytes, as well as their upregulation with lipid accumulation. The distinct time courses observed for the three PAT proteins during FFA exposure might reflect the different roles played by each protein in lipid metabolism in the hepatocyte. Up-regulation of TIP47 and OXPAT might represent an early response to excess lipids, while, in correspondence with a lipid overload, upregulation of ADRP could address lipids towards storage.
\end{abstract}

Correspondence to: Dr Laura Vergani, Department of Biology, Università di Genova, Corso Europa 26, I-16132 Genova, Italy E-mail: laura.vergani@unige.it

Key words: adipose differentiation-related protein, tail-interacting protein of $47 \mathrm{kDa}$, oxidative tissue-enriched PAT protein, primary rat hepatocyte cultures, lipid droplets, fatty liver model

\section{Introduction}

Excess intake of nutrients may cause ectopic fat accumulation in hepatocytes (steatosis) resulting in a condition called nonalcoholic fatty liver disease (NAFLD) $(1,2)$. The pathophysiological basis of NAFLD is excess of circulating free fatty acids (FFAs) that enter the hepatocyte where they are converted to triacylglycerol (TAG) and stored as a form of cytosolic lipid droplets (LDs) (review in ref. 3).

LDs store energy-rich fats for later use as metabolic fuel, but also act as dynamic cell organelles of high complexity connecting storage and traffic of lipids towards specific cellular destinations or signaling pathways (4). Typically, LDs are composed of a core of neutral lipids surrounded by a phospholipid monolayer and a group of proteins of the 'PAT family'. The acronym refers to the first three members identified, perilipin, adipocyte differentiation-related protein (ADRP), and tail interacting protein (TIP47). Two other proteins, S3-12 $(5,6)$ and oxidative tissue-enriched PAT protein (OXPAT) $(7,8)$ have been recently identified in mice and humans $(9)$.

The members of the PAT family show different size, stability, tissue specificity and affinity for LDs, thus suggesting that each PAT protein may be involved in distinct cellular functions $(10,11)$.

In human and mouse liver, ADRP and TIP47 are reported to be constitutively expressed $(11,12)$. In rat liver, ADRP is well documented as a steatosis marker in both NAFLD and alcoholic liver disease (ALD) syndromes, whereas OXPAT and TIP47 are not documented. ADRP (adipophilin) is almost ubiquitously expressed in basal conditions and it is up-regulated in the hepatocytes of patients and mice with fatty liver (13). Lipid accumulation seems to prevent ADRP protein degradation (14). In rat hepatocytes, ADRP promotes the incorporation of lipids in LDs, thus resulting in enlargement of the LD size, and inhibits FFA $\beta$-oxidation (15).

In humans and mice, OXPAT is selectively expressed in tissues that exhibit a high capacity for FFA oxidation, including heart, brown adipose tissue, liver and muscle $(7,16)$. OXPAT is stable in the cytosol of cultured cells in the absence of excess lipids, but it moves to the LD surface during lipid loading (9). Therefore, OXPAT is postulated to regulate lipid storage for short-term utilization through oxidative pathways (8).

TIP47, also known as placental protein 17 (PP17), shares a very high degree of homology with ADRP. Like OXPAT, TIP47 binds LDs in response to lipid loading (17). 
Peroxisome proliferator-activated receptors (PPARs) are transcription factors regulating expression of many proteins involved in lipid metabolism. Expression of the PPARs $(\alpha, \gamma$ and $B / \delta$ ) generally correlates well with the tissue expression profile of the PAT proteins. In human hepatocytes, PPAR $\gamma$ is documented to regulate both ADRP and OXPAT expression $(8,13)$. By contrast, TIP47 is not regulated by PPARs $(18)$.

The present study was aimed at assessing if OXPAT and TIP47 are constitutively expressed in adult rat hepatocytes. Moreover, possible modulation of ADRP, OXPAT and TIP47 expression by lipid loading was evaluated. To this aim, fatenriched hepatocytes were obtained by exposure of primary rat hepatocytes to a mixture of FFAs for different times, according to the model of cellular steatosis previously described in human hepatoma HepG2 cells (19).

In fat-enriched hepatocytes, the increase in TAG content was paralleled by the increase in size and number of LDs. Moreover, the reported lipid accumulation was associated with up-regulation of ADRP, OXPAT and TIP47, despite distinct time courses in relation of exposure to FFAs and lipid accumulation. These findings support the hypothesis that expression of these PAT proteins represents selective markers of fat accumulation also in rat liver.

\section{Materials and methods}

Chemicals. All chemicals, unless otherwise indicated, were of analytical grade and were obtained from Sigma-Aldrich Corp. (Milan, Italy).

Primary rat hepatocyte isolation and culture. Hepatocytes were isolated from adult male Wistar rats (Harlan-Italy, S. Pietro al Natisone, Italy), as previously described (20). Cells suspended in serum-free Dulbecco's modified Eagle's medium (DMEM) supplemented with essential and non-essential amino acids were plated onto collagen-coated tissue culture dishes in the presence of insulin $(0.1 \mathrm{U} / \mathrm{ml})$ and dexamethasone $\left(10^{-6} \mathrm{M}\right)$. After $90 \mathrm{~min}$, the medium was replaced by fresh DMEM containing $1 \%$ bovine serum albumin (DMEM-1\%BSA); cells were further incubated at $37^{\circ} \mathrm{C}$ in a humidified atmosphere of $5 \% \mathrm{CO}_{2}$ in air for $24 \mathrm{~h}$ (19). To induce lipid accumulation, hepatocytes were incubated in the presence of the long-chain FFAs oleate and palmitate (1.5 mM final concentration, 2:1 molar ratio) for 12, 24 and $36 \mathrm{~h}$ (19). Control hepatocytes were incubated in the medium without addition of FFAs for the same periods of time. In all experiments, cell viability, estimated by trypan blue exclusion test, was $>90 \%$. At the end of treatment, cells were scraped, washed twice in sodium phosphate buffer (PBS) and then stored at $-80^{\circ} \mathrm{C}$ until use.

For microscopical analysis, hepatocytes were grown and treated directly on collagen-coated glass slides (Falcon, BD, Milan, Italy).

Lipid quantification. Intracellular triacylglycerol (TAG) content in was quantified after lysis of hepatocytes by spectrophotometric analysis using the commercial GPO-PAP kit (Roche, Milan, Italy), according to the manufacturer's instructions. At the indicated times, cells were washed, scraped and briefly centrifuged; the pellet was resuspended in $200 \mu 1$ methanol. Lipids were extracted by adding $500 \mu 1$
Table I. Oligonucleotide primers used for real-time RT-PCR experiments.

\begin{tabular}{llr}
\hline Gene name & Forward primer (5'-3') & Reverse primer (5'-3') \\
\hline GAPDH & GACCCCTTCAT & CGCTCCTGGAAG \\
& TGACCTCAAC & ATGGTGATGGG \\
PPAR- $\gamma$ & CGGAGTCCTCCC & GGCTCATATCTG \\
& AGCTGTTCGCC & TCTCCGTCTTC \\
ADRP & CCGAGCGTGG & GAGGTCACGGT \\
& TGACGAGGG & CCTCACTCCC \\
OXPAT & GGATGTCCGG & GTGCACGTGG \\
& TGATCAGAC & CCCTGACCAG \\
TIP47 & GGAACTGGTG & GGTCACATCC \\
& TCATCAACAG & ACTGCTCCTG \\
\hline
\end{tabular}

chloroform and the resulting mixture was shaken for $1 \mathrm{~h}$. After addition of $250 \mu 1 \mathrm{H}_{2} \mathrm{O}$ and brief vortexing, samples were centrifuged at $2,000 \mathrm{x}$ g for $25 \mathrm{~min}$. The lower phase was collected and evaporated overnight. The dry pellet was then incubated with $500 \mu 1$ of the Roche solution at $37^{\circ} \mathrm{C}$ for $15 \mathrm{~min}$. TAG content was estimated by recording the absorbance at $500 \mathrm{~nm}$ in a Varian Cary 50 spectrophotometer (Varian, Torino, Italy). Values were normalized for the protein content $(\mathrm{mg} / \mathrm{ml})$ determined by the bicinchoninic acid (BCA) method using bovine serum albumin (BSA) as a standard (21). Data are expressed as percent TAG content relative to controls.

Neutral lipid staining. In intact cells, neutral lipids were visualized using the soluble selective dye oil red O (ORO) (22). After treatments, slides were washed three times with iced PBS and fixed with 4\% paraformaldehyde in PBS (pH 7.4) at $4^{\circ} \mathrm{C}$ for $1 \mathrm{~h}$, then incubated in $0.3 \%$ working ORO solution (prepared from a stock solution of $0.5 \%$ ORO in $60 \%$ triethylphosphate) at room temperature for $30 \mathrm{~min}$. ORO-stained cells were then counterstained with hematoxylin and slides were mounted in $10 \%$ glycerol in $10 \mathrm{mM}$ Tris- $\mathrm{HCl}$. Slides were examined by digitized optical microscopy using light microscope Nikon Eclipse E80i (Nikon, Japan) equipped with x10 objective lens. Densitometric analysis of ORO-stained cells was performed with a custom-made routine on the Optimas 6.5 image analysis system (Optimas, Washington DC) by extracting the mean $\log$ inverse value of pixels within the area boundary (mArLIGV).

RNA extraction and real-time RT-PCR. Total RNA was isolated from different hepatocyte samples by the acid phenol-chloroform procedure using the Trizol reagent (Sigma) according to the manufacturer's instructions (23). First strand cDNA was synthesized from $1 \mu \mathrm{g}$ of total RNA (24). Quantitative RT-PCR was performed in quadruplicate in a final volume of $25 \mu \mathrm{l}$ containing $0.3 \mu \mathrm{M}$ of each primer, $10 \mathrm{ng}$ cDNA, 1x SybrGreen PCR Master Mix and were analyzed in 96-well optical reaction by 7900 HT fast real-time PCR system (Applied Biosystems, Monza, Italy). Primers for the genes of interest (Table I) were designed ad hoc starting from the coding sequences of Rattus norvegicus (http://www.ncbi.nlm.nih.gov/Genbank/GenbankSearch.htm) 
A

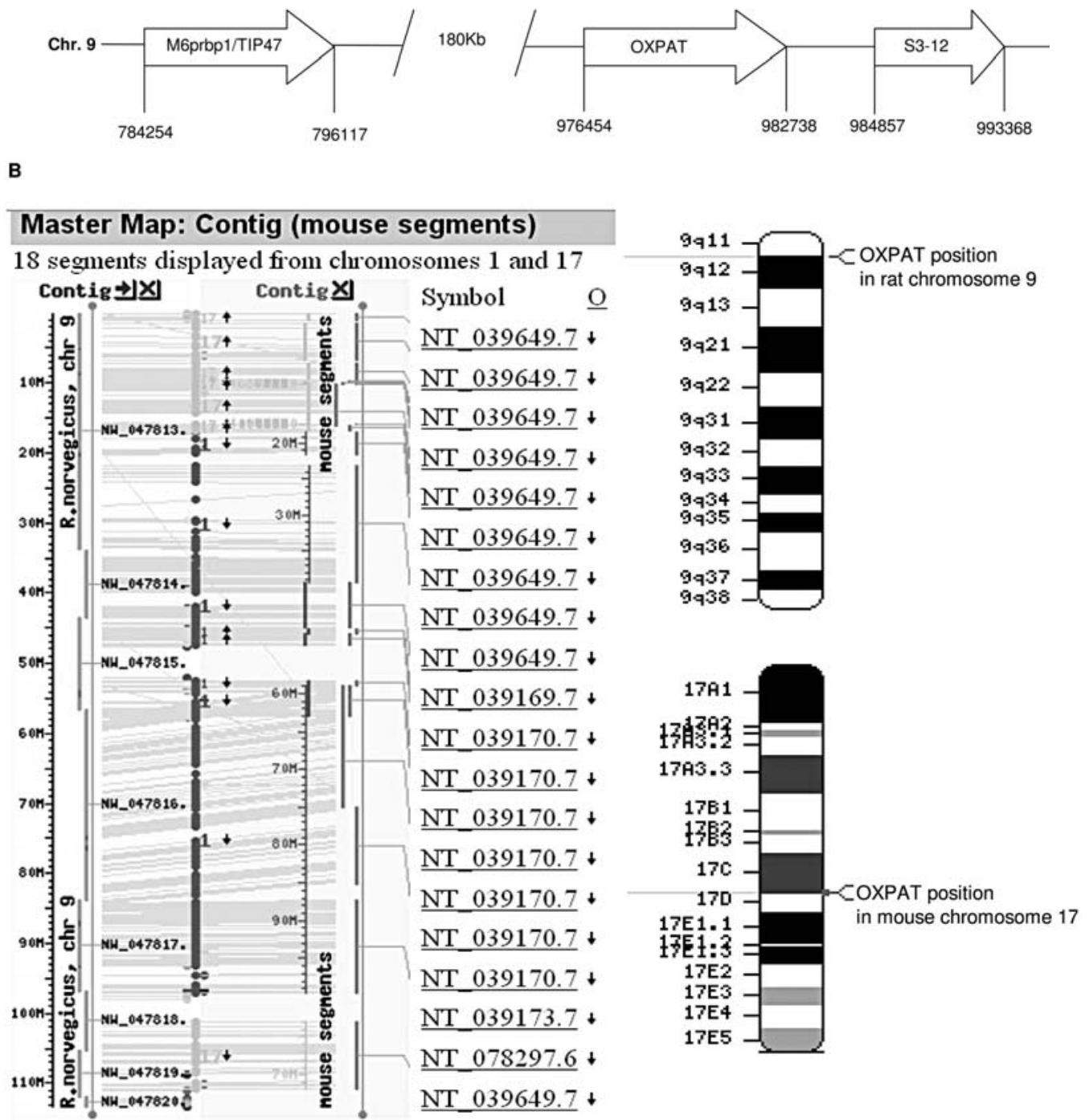

Figure 1. OXPAT and TIP47 position in the rat and mouse genome. (A) Tip47, Oxpat and S3-12 are located on rat chromosome 9. As in other mammalian genomes, Oxpat and S3-12 are encoded by tandem genes, separated by 2119 bp. Tip47 is located $180 \mathrm{kbp}$ upstream. (B) Left, Mapview/Contig rat chromosome alignment (http://www.ncbi.nlm.nih.gov/projects/mapview/) against the whole mouse genome. The match of rat chromosome 9 with mouse chromosome 17 and chromosome 1 is also shown. Right, OXPAT location in rat chromosome 9 (9q11) and in mouse chromosome 17.

and synthesized by TibMolBiol custom oligosynthesis service (Genova, Italy). The thermal protocol consisted of 3 min initial denaturation at $95^{\circ} \mathrm{C}$, followed by 40 cycles of amplification. The relative quantity of target mRNA was calculated by using the comparative $\mathrm{C}_{\mathrm{T}}$ method and normalized for the expression of glyceraldehyde 3-phosphate dehydrogenase (GAPDH). Parallel measurements were performed using actin and $18 \mathrm{~S}$ rRNA as housekeeping genes without observing any significant difference in the expression profiles. The normalized expression was expressed as relative quantity of mRNA (fold induction) with respect to controls (25).

Statistical analysis. Data on real-time RT-PCR are means \pm SD of at least three independent experiments performed in triplicate. Data on TAG quantification are means \pm SD of three independent experiments. Statistical analysis was performed by using ANOVA followed by Bonferroni post hoc test (Instat software, GraphPad Software, Inc., San Diego, CA, USA).

\section{Results}

Identification of OXPAT and TIP47 in rat genome and constitutive transcription in hepatocytes. A coding sequence annotated as 'predicted to be similar to lipid protein associated' (ncbi ID, 501283) was identified in the genome of Rattus norvegicus by blasting the cDNA sequence of the Oxpat derived from Mus musculus (ncbi ID, DQ473305). Alignment of this rat sequence against the Oxpat sequences derived from different mammalian sources revealed high identity values ranging from 69.7 to $90.1 \%$ (data not shown). Based on these results, we could refer to this cDNA sequence as the sequence encoding for OXPAT in genome of Rattus norvegicus.

In rat genome, the Oxpat gene is adjacent to a sequence encoding the PAT protein S3-12, and about $180 \mathrm{kbp}$ downstream of the M6prbpl gene encoding the TIP47 protein (ncbi ID, NW047865.1) (Fig. 1A). This location is similar to that of the mouse, in which the Oxpat gene is located on 
A \% IDENTITY for PAT proteins

\begin{tabular}{|c|c|cc|}
\hline & ADRP & TIP47 & OXPAT \\
\hline ADRP & & 29.1 & 38.9 \\
TIP47 & & & 30.4 \\
\cline { 3 - 4 } OXPAT & & & \\
\hline
\end{tabular}

ADRP

TIP 47

OXPAT

ADRP

TIP47

OXPAT

ADRP

TIP 47

OXPAT

ADRP

TIP 47

OXPAT

ADRP

TIP 47

OXPAT

ADRP

TIP 47

OXPAT

ADRP

TIP47

OXPAT

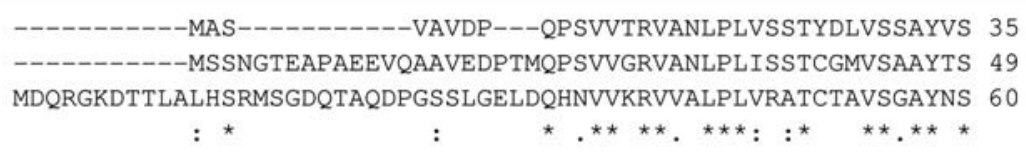

TKDQHPYLRSVCEMAEKGVRTVTSVAVTGALPI IQKLEPQIAVANTYACKGLDRMEARLP 95 TKESYPHVRTVCDVAEKGVKTLTTAAVSRAQPILSKLEPQIVTANKYAHQGLDRLQESLP 109 AKDRHPLLGSACRFAEHCVCSVATCALDHAQPLLEHLQPKLATVNDLACRGLDKLEEKLP 120

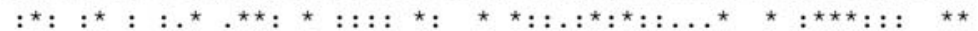

ILNQPTSEIVAN----ARGAVTGAKDVVTTTMAGAKDSVASTVSGVVDKTKGAVTGSVER 151 ILQQPTEKVLADTKELVSSTVSGAREMVSSSVSGAKDTVATRVTGAVDVTCGAVKSSVDM 169 FLQQPSDTVVTS-------------------AKDAVAKSVTGVVDLAQRGRRWSGEL 158 $:{ }^{\star}:{ }^{\star \star}: .::: .{ }^{\star \star \star}:{ }^{\star \star} .{ }^{\star}:{ }^{\star},{ }^{\star \star}: .{ }^{\star}:$

TKSVVNGGIDTVLG--MVQLMSSGVENAISKSELLVDQYLPLTQKELEMEAKKVEGFDMV 209 TKSAMTSGVQSIMGSRVGQMVISGVDRVLVKSEAWADNRLPLTDTELALIATSPEGADMA 229 RRSVS-------------QAMDTVLGKSEELVDHFLPMTEAELVALATESQGPEVG 201

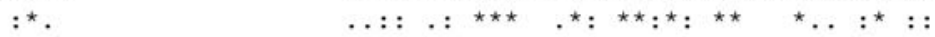

QKQR------YYERLESLSTKICTRAYHQALGRIKDAKQKGQETISQLHSTVHLIEFARK 263 SLQQQRQERSYFVRLGSLSERLRNQAYEHSLGKLCNVRQSAQDALQQLAHVLSLMESVKQ 289 SVEEQRQKQGYFVRLGSLSARLRHLAYQHSLGKLRESKHRTQEMLAQLQKTLELIQHMQS 261

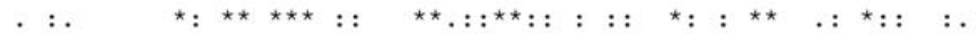

NVHSANQKIQDKLSVSWVEWKRIVGYDDTDESHCAEHIESHTLSMARNLTQQLHTTCQTL 323 GVDQRLEEGQEKLHQMWLTWNQKTPQDAEKDPSKPEQVEAQALSVFRDIAQQLQSTCVAL 349 RASPTPTFHHPKVQELCGDWS-----PCLENGYRHSQVELETLALSRSLTLELQSAVDAL 316

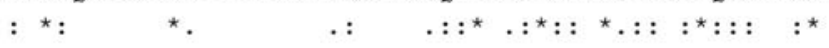

LFNVQGLPQNIQDQAKHLGVMAGDIYSAFRNVTSFKEVSDGVLTSSKGQLQKMKESLDEV 383 GASIQGLPSHVREQAQQARSQVDDLQATFSGIHSFQDLTASVLAQTREHIARARDALEHT 409 AGCVRGLPPSAQAKVAEVQRSVDALQATFADAHCLGDVAPTALAEGQDSVAQAHACVDEF 376

$$
:: \star \star \star \quad:: . . \quad \ldots:::^{\star} . \quad .::::{ }^{\star}: .: \text { : : : } .:: .
$$

\begin{tabular}{|c|c|c|c|}
\hline ADRP & MDYLVI & NTPLNWLVGPFYPQS & 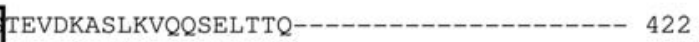 \\
\hline TIP47 & VEYVAC & NTPAMWLVGPFAPGI & -18 \\
\hline OXPAT & $\begin{array}{l}\text { LDLVLF } \\
:::\end{array}$ & $\begin{array}{l}\text { AMPLAWLVGPFAPII } \\
\star \star \star \star \star \star \star \star \star \star\end{array}$ & $\begin{array}{l}\text { VERSEPLINLATCVDEVVGDPDPRWAHMDWPAQQRAWEA } 436 \\
\text { * }\end{array}$ \\
\hline
\end{tabular}

ADRP

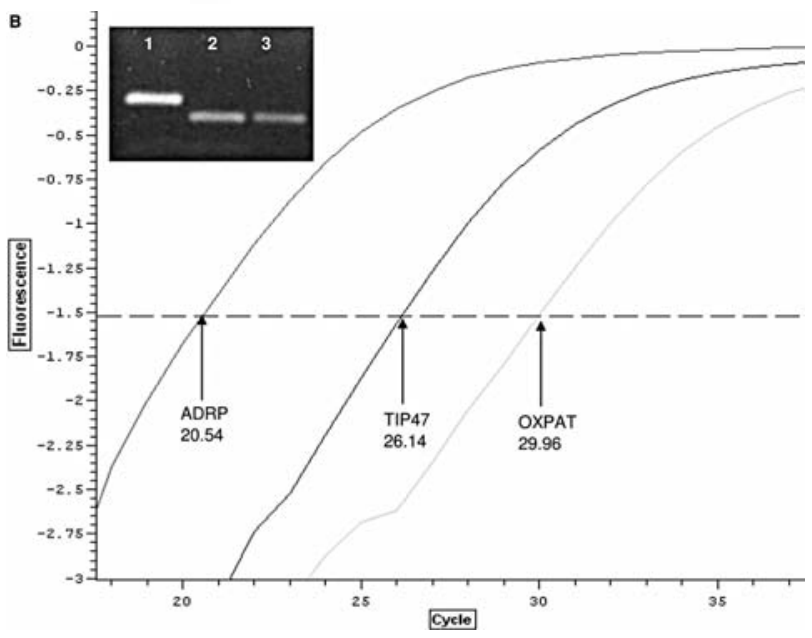

Figure 2. Constitutive transcription of ADRP, OXPAT and TIP47 in rat hepatocytes. (A) The amino acid sequences of rat ADRP, OXPAT and TIP47 aligned by Clustal W2 method. Similarity in each position is symbolized in the lower line. "Identical residues, :similar residues, and ${ }^{\text {different residues }}$ are labeled. At the bottom, the matrix shows the similarity among the three PAT proteins. The black box underlines the carboxy-terminal motif conserved in all PAT proteins, except perilipin. (B) Constitutive relative abundance of the three PAT protein transcripts in rat hepatocytes evaluated by real-time RT-PCR. Amplification curves of ADRP, TIP47 and OXPAT are shown with a crossing point at $\sim 20,26$ and 30 cycles, respectively. $x$-axis, amplification cycle number; $y$-axis, normalized fluorescence signal. 
chromosome 17. In contrast, in the rat, the Oxpat gene maps to chromosome 9 (9q11). The whole chromosome alignment of rat chromosome 9 against the whole mouse genome indicates that the two terminal regions of rat chromosome 9 overlap mouse chromosome 17, while the majority of rat chromosome 9 corresponds to mouse chromosome 1 (Fig. 1B).

Fig. 2A shows the alignment and the sequence identity for the three PAT proteins from Rattus norvegicus. The alignment of OXPAT, TIP47 and ADRP protein sequences revealed high scores of identity, ranging from 29.1 to $38.2 \%$. The carboxy-terminal motif, highly conserved in all PAT proteins, is indicated by a black box (26).

Expression of the three PAT proteins in rat hepatocytes was quantified by real-time RT-PCR (Fig. 2B). In control hepatocytes, the amplification curves showed differences in $\Delta \mathrm{Ct}$ values over five cycles between ADRP and TIP47, and over nine cycles between ADRP and OXPAT. This observation points at the following relative abundance of PAT protein mRNAs in adult rat hepatocytes, ADRP $>$ TIP47>OXPAT.

Lipid accumulation and PPAR $\mathrm{mRNA}$ expression. Fig. 3 shows the time course of lipid accumulation in primary adult rat hepatocytes exposed to the oleate/palmitate mixture (final concentration 1.5 mM). Exposure to FFAs for 12, 24 and $36 \mathrm{~h}$ raised the TAG content by $23 \%(\mathrm{p}<0.05), 73 \%(\mathrm{p}<0.001)$, and $166 \%,(p<0.001)$, respectively, with respect to controls (C) (Fig. 3A). No lipotoxicity was observed when cell viability was checked by Trypan blue test.

Fig. 3B displays representative images of neutral lipid accumulation evaluated by the ORO staining. In hepatocytes incubated with (FFAs) or without (C) the oleate/palmitate mixture, lipid accumulation was associated with the appearance of red-stained LDs that increased in size and number with the time of exposure.

Therefore, the results indicate that the protocol of exposure of primary rat hepatocytes to the oleate/palmitate mixture results in fat-enriched hepatocytes with the degree of fat accumulation being roughly proportional to the time of exposure.

Murine models developing fatty livers usually express enhanced levels of PPAR $\gamma$. When expression of PPAR $\gamma$ was assessed by RT-PCR, the results show up-regulation of PPAR $\gamma$ at $12 \mathrm{~h}$ exposure to FFAs (3.8-fold induction with respect to control, p<0.001) (Fig. 3C). At longer exposure times, PPAR $\gamma$ expression declined to 1.5 -fold induction at $24 \mathrm{~h}(\mathrm{p}<0.05)$ and returned to control values at $36 \mathrm{~h}$.

Expression of PAT proteins. Expression of OXPAT, TIP47 and ADRP mRNA in different experimental conditions was quantified by real-time RT-PCR (Fig. 4). Early after exposure of hepatocytes to FFAs, OXPAT transcription showed marked up-regulation with respect to control (1.75-fold induction at $12 \mathrm{~h} ; \mathrm{p}<0.001)$ that persisted at longer times of exposure (1.71- and 1.53-fold induction at 24 and $36 \mathrm{~h} ; \mathrm{p}<0.001$ and $\mathrm{p}<0.01$, respectively).

In rat hepatocytes, also TIP47 mRNA expression was upregulated after $12 \mathrm{~h}$ exposure to FFAs (1.42-fold induction with respect to control; $\mathrm{p}<0.01$ ), followed by a slow return to control values at longer times of exposure.

On the other hand, after exposure to FFAs the ADRP mRNA expression increased significantly with respect to control only at longer times (1.37- and 1.28-fold induction at 24 and $36 \mathrm{~h} ; \mathrm{p}<0.001$ and $\mathrm{p}<0.01$, respectively).

\section{Discussion}

Five members of the PAT family proteins, each with characteristic tissue-specificity and affinity, have been identified in LDs of different tissues including muscle, liver, brown and white adipose tissue (10). In the liver of humans and mice, three major PAT proteins are commonly expressed, ADRP, OXPAT and TIP47 (9). By contrast, little information is so far available on PAT proteins in the rat liver, where only ADRP expression has been reported (27).

In the present study, we identified, for the first time, the presence in adult rat hepatocytes of OXPAT and TIP47 mRNA transcripts. Moreover, our data show that in rat hepatocytes the constitutive expression of OXPAT is higher than that of TIP47, and both genes show a lower basal expression with respect to ADRP. The higher constitutive expression of ADRP with respect to the other two PAT proteins in rat hepatocytes confirm previous reports obtained in vivo in mouse liver (16).

An interesting difference between the mouse and the rat is the chromosomal location of the pertinent genes. While in the rat, Oxpat, Tip47 and S3-12 genes map on chromosome 9, in mouse the same genes are located on chromosome 17. However, chromosome alignment indicates that the two terminal regions of rat chromosome 9 overlap mouse chromosome 17 , while the majority of rat chromosome 9 corresponds to mouse chromosome 1 . It is noteworthy that rat chromosome 9 scored the highest recombination rate (0.71) compared with mouse chromosomes 1 and 17 (0.61 and 0.57 , respectively) (28), and that the rat genome has an higher number of chromosome arms (64) with respect to mouse genome (40) (29). These data, together with the telomeric position of the Oxpat gene in rat, suggest a highest probability that recombination events occurred in rat genome rather than in mouse. However, recombination is known to occur non-uniformly across the genomes of mammals (30-33), and we are far from understanding how and why it takes place during evolution.

Moreover, in the rat genome, the Oxpat gene is located immediately upstream of the gene encoding S3-12, and close to the Tip47 gene. We also found this genomic organization in Homo sapiens, Canis familiaris and Bos Taurus. Dalen and coworkers (16) hypothesized that the S3-12 and Oxpat genes were derived from gene duplication of an ancestral version of the Tip47 gene, since their transcripts are closely related. The present study confirms this genomic organization also in Rattus norvegicus.

In the liver, lipid accumulation occurs in several pathophysiological conditions, such as NAFLD and ALD. In mammals, fatty liver results in up-regulation of the expression of one or more PAT proteins. In this study, we investigated possible changes in the mRNA level of three PAT proteins as a function of lipid accumulation in the hepatocyte. Primary cultures of rat hepatocytes were exposed to a mixture of long-chain FFAs for increasing periods of time in order to mimic different degrees of hepatic 'steatosis'. According to previous reports, the FFA mixture contained a low proportion of the saturated with respect to the mono-unsaturated FFA 


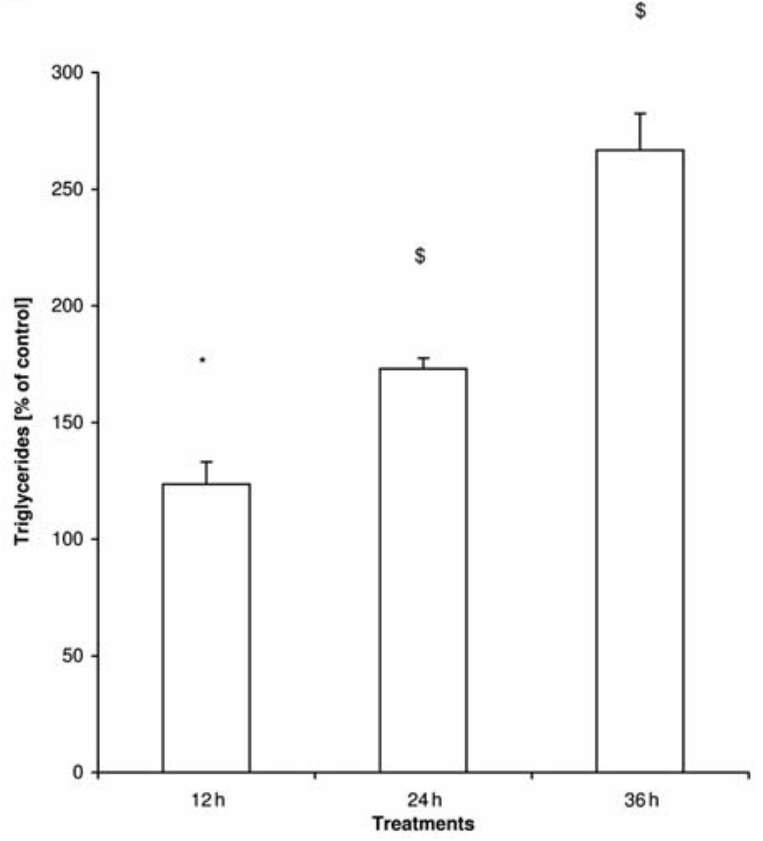

B

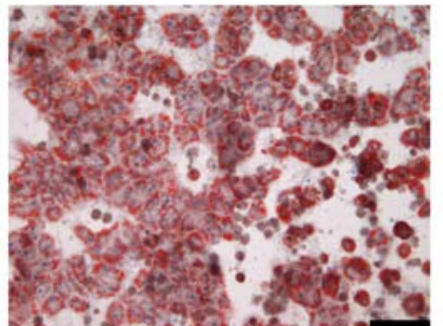

FFAs $12 \mathrm{~h}$

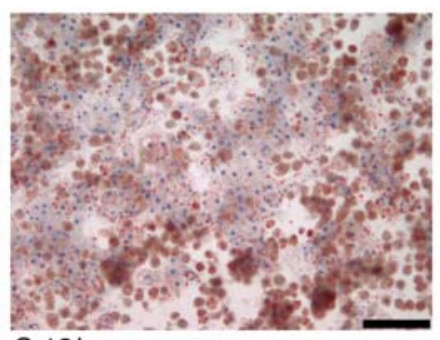

C $12 \mathrm{~h}$

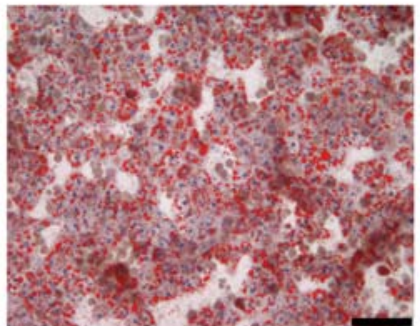

FFAs $24 \mathrm{~h}$

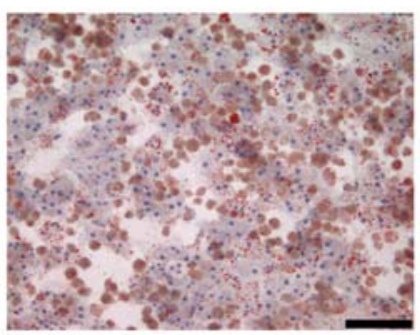

$\mathrm{C} 24 \mathrm{~h}$

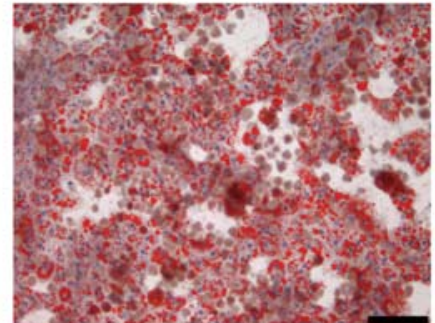

FFAs $36 \mathrm{~h}$

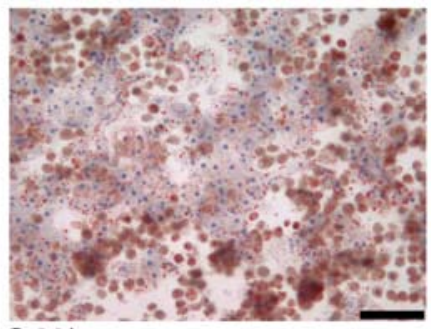

$\mathrm{C} 36 \mathrm{~h}$ s

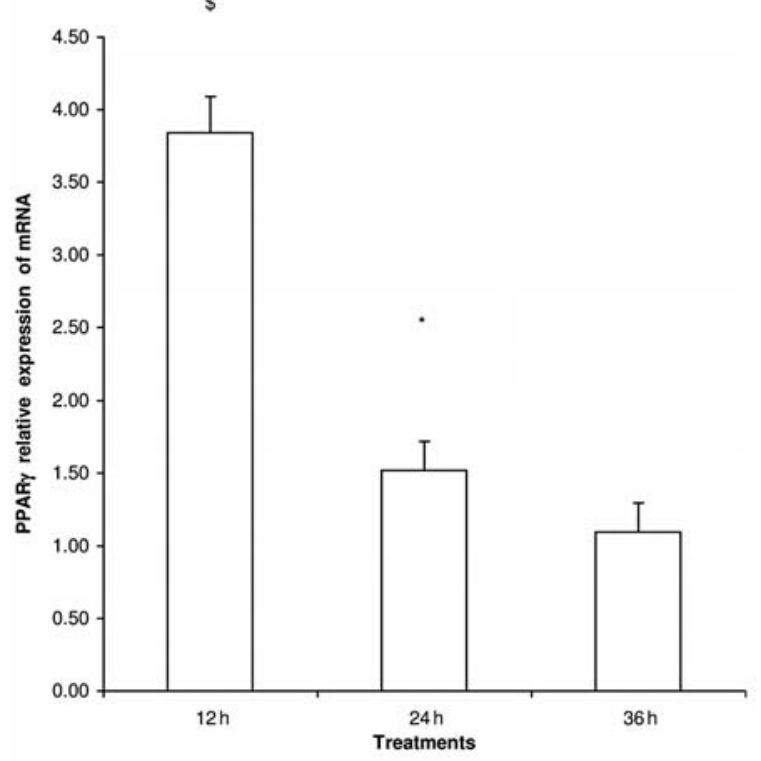

Figure 3. Effects of exposure to FFAs on lipid accumulation and PPAR $\gamma$ mRNA expression. (A) Spectrophotometric quantification of triacylglycerol (TAG) in hepatocytes incubated with the oleate/palmitate mixture (molar ratio 2 : 1 ; final concentration $1.5 \mathrm{mM}$ ) for 12,24 and $36 \mathrm{~h}$. Data (mean $\pm \mathrm{SD}$ ) are expressed as percentage of the corresponding controls (hepatocytes incubated in the medium without addition of FFAs for the same periods of time). ${ }^{*} \mathrm{p}<0.05 ;{ }^{\$} \mathrm{p}<0.001$. Data are means of three independent experiments. (B) Representative images of ORO-stained hepatocytes incubated with (FFAs) and without (C) the oleate/palmitate mixture for 12, 24 and $36 \mathrm{~h}$. Nuclear staining with hematoxylin (magnification, x10; bar, $100 \mu \mathrm{m}$ ). (C) PPAR $\gamma$ mRNA expression in hepatocytes exposed to the oleate/palmitate mixture for 12,24 and $36 \mathrm{~h}$. Data (mean $\pm \mathrm{SD}$ ) are expressed as fold induction with respect to the corresponding controls (hepatocytes incubated in the medium without addition of FFAs for the same periods of time) after normalization for GAPDH mRNA. ${ }^{\$} \mathrm{p}<0.001,{ }^{*} \mathrm{p}<0.05$. Data are means of three independent experiments performed in triplicate. 


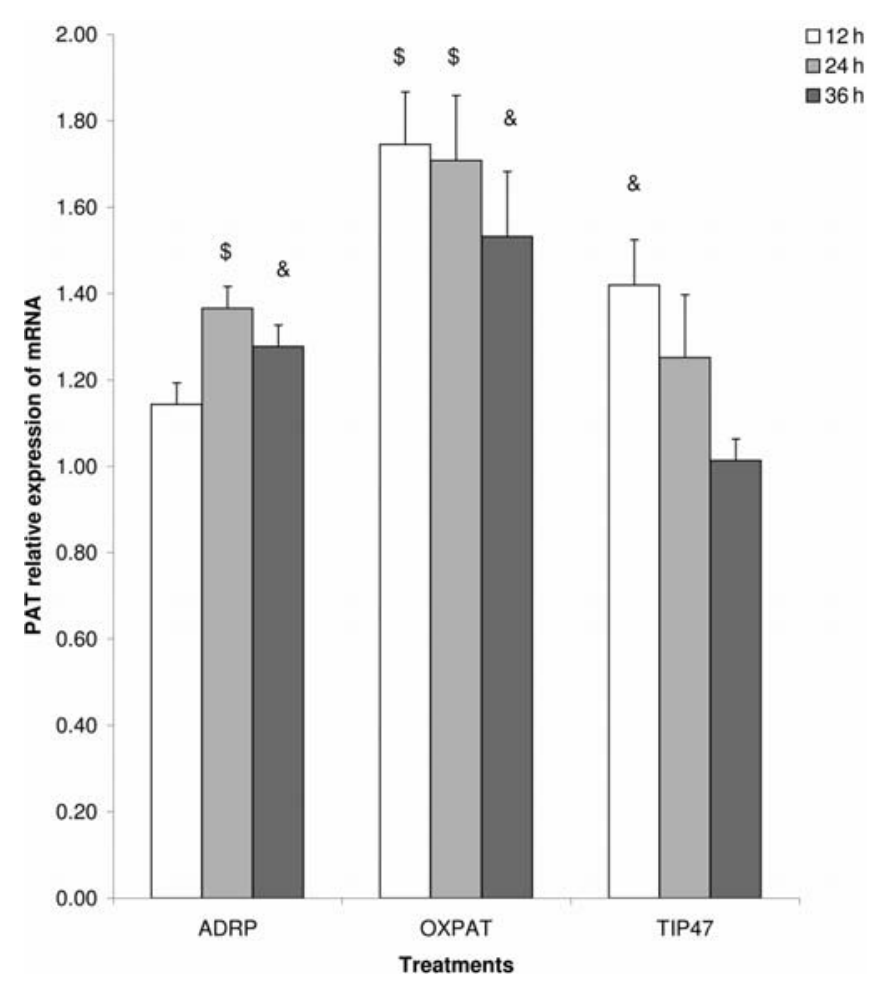

Figure 4. Effects of exposure to FFAs on mRNA expression of PAT proteins. PAT protein transcription in hepatocytes exposed the oleate/palmitate mixture for 12, 24 and $36 \mathrm{~h}$. Data (mean $\pm \mathrm{SD}$ ) are expressed as fold induction with respect to the corresponding controls (hepatocytes incubated in the medium without addition of FFAs for the same periods of time) after normalization for GAPDH mRNA. ${ }^{\&} \mathrm{p}<0.01,{ }^{\$} \mathrm{p}<0.001$. Data are means of three independent experiments performed in triplicate.

(oleate/palmitate $2: 1$ ratio, total concentration $1.5 \mathrm{mM}$ ) to minimize possible cytotoxic effects $(34,35)$; moreover, this FFA concentration is similar to the physiological FFA levels of mammalian plasma that usually range between 0.2-2 mM.

The results evidenced a time-dependent increase in lipid accumulation that appeared as micro- and macro-vesicles in the cytosol. Overall, in our in vitro model, the longer the time of exposure to FFAs, the greater the amount of lipids accumulated in the cells.

The pivotal role of PPAR $\gamma$ in the liver, although important for the regulation of genes involved in lipid metabolism, has generally not been fully appreciated, due to the fact that PPAR $\gamma$, in contrast to PPAR $\alpha$ or PPAR $\delta$, is not abundantly expressed in liver under normal conditions. However, recent findings have revealed that in obese and mice fed a high fat diet, PPAR $\gamma$ mRNA is highly up-regulated in the liver $(36,37)$. Our results indicate that, also in vitro, hepatic lipid accumulation is accompanied by significant up-regulation of PPAR $\gamma$ expression, which peaked at early times ( $12 \mathrm{~h}$ exposure in our case) and decreased thereafter. This suggests that PPAR $\gamma$ expression is up-regulated at inception of lipid accumulation, but that excessive lipid loading, obtained by prolonging exposure to FFAs, reverses this effect.

Interestingly, in rat hepatocytes, the transcription profiles of the different PAT proteins displayed remarkably distinct time-courses during FFA exposure. Both OXPAT and TIP47 were significantly up-regulated at $12 \mathrm{~h}$, in correspondence to the first increase in lipid accumulation, but the increase in OXPAT expression was persistent, whereas that of TIP47 was transient, declining towards control values at high lipid overload. On the contrary, ADRP expression reached maximum values in correspondence to a large lipid overload. These data resemble those obtained in oleate-treated murine adipocytes, where TIP47 and OXPAT were shown to coat nascent LDs during rapid fat storage, whereas ADRP was involved in sustained fat storage (38). Moreover, the plateau observed for ADRP expression at $24 \mathrm{~h}$ of FFA exposure is very similar to what was observed in HepG2 cells incubated with troglitazone by Motomura and coworkers (13).

Such a pattern of PAT expression may reflect the different roles played by each protein in lipid metabolism in the hepatocyte. OXPAT seems to promote FFA consumption and facilitate lipid storage for near-term utilization through oxidative pathways (8). TIP47 mediates delivery of nascent TAG to the storage depots $(8,38)$. On the contrary, ADRP overexpression is associated with increase in size of LDs and inhibition of $B$-oxidation of FFAs as a response of a massive increase in TAG content (15).

Taken together, our data suggest that, after exposure to FFAs, rat hepatocytes initially activate catabolic pathways in an attempt to eliminate the excess of FFAs (i.e. through induction of OXPAT and TIP47 proteins). Then, when the cell is overloaded with lipids, a sustained up-regulation of ADRP would stimulate the fat packaging inside LDs in order to avoid excess stimulation of catabolic pathways that would result in severe oxidative stress condition. Therefore, this study demonstrates that the pattern of PAT protein expression is correlated with the extent of lipid accumulation in hepatocytes as a function of their specific role in lipid metabolism. It is suggested that the level of PAT protein expression is a reliable marker for liver 'steatosis'.

Moreover, the fat-enriched rat hepatocytes described in this study could represent a viable in vitro model of hepatic steatosis that could find numerous applications i.e. to test novel therapeutic strategies for human fatty liver disease.

\section{Acknowledgements}

Special thanks to Mr. Valter Capicchioni for his helpful technical assistance in hepatocyte preparation and to Dr Torelli (Roche, Milan Italy) for his gift of the GPO-PAP kit for triglycerides determination. This work was supported by research grants from MIUR (PRIN 2006), from Compagnia San Paolo (Torino) and from the University of Genova.

\section{References}

1. Adams LA and Angulo P: Treatment of non-alcoholic fatty liver disease. Postgrad Med J 82: 315-322, 2006.

2. Adams LA and Angulo P: Recent concepts in non-alcoholic fatty liver disease. Diabet Med 22: 1129-1133, 2005.

3. Bradbury MW: Lipid metabolism and liver inflammation. I. Hepatic fatty acid uptake: possible role in steatosis. Am J Physiol Gastrointest Liver Physiol 290: G194-G198, 2006.

4. Olofsson SO, Bostrom P, Andersson L, Rutberg M, Perman J and Boren J: Lipid droplets as dynamic organelles connecting storage and efflux of lipids. Biochim Biophys Acta 1791: 448-458, 2008.

5. Scherer PE, Bickel PE, Kotler M and Lodish HF: Cloning of cell-specific secreted and surface proteins by subtractive antibody screening. Nat Biotechnol 16: 581-586, 1998. 
6. Wolins NE, Skinner JR, Schoenfish MJ, Tzekov A, Bensch KG and Bickel PE: Adipocyte protein S3-12 coats nascent lipid droplets. J Biol Chem 278: 37713-37721, 2003.

7. Yamaguchi T, Matsushita S, Motojima K, Hirose F and Osumi T: MLDP, a novel PAT family protein localized to lipid droplets and enriched in the heart, is regulated by peroxisome proliferatoractivated receptor alpha. J Biol Chem 281: 14232-14240, 2006.

8. Wolins NE, Quaynor BK, Skinner JR, et al: OXPAT/PAT-1 is a PPAR-induced lipid droplet protein that promotes fatty acid utilization. Diabetes 55: 3418-3428, 2006.

9. Bickel PE, Tansey JT and Welte MA: PAT proteins, an ancient family of lipid droplet proteins that regulate cellular lipid stores. Biochim Biophys Acta 1791: 419-440, 2009.

10. Brasaemle DL: Thematic review series: adipocyte biology. The perilipin family of structural lipid droplet proteins: stabilization of lipid droplets and control of lipolysis. J Lipid Res 48: 2547-2559, 2007.

11. Ducharme NA and Bickel PE: Lipid droplets in lipogenesis and lipolysis. Endocrinology 149: 942-949, 2008.

12. Brasaemle DL, Barber T, Wolins NE, Serrero G, BlanchetteMackie EJ and Londos C: Adipose differentiation-related protein is an ubiquitously expressed lipid storage dropletassociated protein. J Lipid Res 38: 2249-2263, 1997.

13. Motomura W, Inoue M, Ohtake T, et al: Up-regulation of ADRP in fatty liver in human and liver steatosis in mice fed with high fat diet. Biochem Biophys Res Commun 340: 1111-1118, 2006.

14. Londos C, Sztalryd C, Tansey JT and Kimmel AR: Role of PAT proteins in lipid metabolism. Biochimie 87: 45-49, 2005.

15. Magnusson B, Asp L, Bostrom P, et al: Adipocyte differentiationrelated protein promotes fatty acid storage in cytosolic triglycerides and inhibits secretion of very low-density lipoproteins. Arterioscler Thromb Vasc Biol 26: 1566-1571, 2006.

16. Dalen KT, Dahl T, Holter E, et al: LSDP5 is a PAT protein specifically expressed in fatty acid oxidizing tissues. Biochim Biophys Acta 1771: 210-227, 2007.

17. Wolins NE, Rubin B and Brasaemle DL: TIP47 associates with lipid droplets. J Biol Chem 276: 5101-5108, 2001.

18. Dalen KT, Schoonjans K, Ulven SM, et al: Adipose tissue expression of the lipid droplet-associating proteins S3-12 and perilipin is controlled by peroxisome proliferator-activated receptor-gamma. Diabetes 53: 1243-1252, 2004.

19. Feldstein AE, Werneburg NW, Canbay A, et al: Free fatty acids promote hepatic lipotoxicity by stimulating TNF-alpha expression via a lysosomal pathway. Hepatology 40: 185-194, 2004.

20. Fugassa E, Gallo G, Voci A and Cordone A: RNA synthesis in primary cultures of adult rat hepatocytes. In Vitro 19: 299-306, 1983.

21. Wiechelman KJ, Braun RD and Fitzpatrick JD: Investigation of the bicinchoninic acid protein assay: identification of the groups responsible for color formation. Anal Biochem 175: 231-237, 1988.

22. Koopman R, Schaart G and Hesselink MK: Optimisation of oil red $\mathrm{O}$ staining permits combination with immunofluorescence and automated quantification of lipids. Histochem Cell Biol 116: 63-68, 2001.
23. Chomczynski P and Sacchi N: Single-step method of RNA isolation by acid guanidinium thiocyanate-phenol-chloroform extraction. Anal Biochem 162: 156-159, 1987.

24. Grasselli E, Canesi L, Voci A, et al: Effects of 3,5-diiodo-Lthyronine administration on the liver of high fat diet-fed rats. Exp Biol Med (Maywood) 233: 549-557, 2008.

25. Pfaffl MW: A new mathematical model for relative quantification in real-time RT-PCR. Nucleic Acids Res 29: E45, 2001.

26. Thompson JD, Higgins DG and Gibson TJ: CLUSTAL W: improving the sensitivity of progressive multiple sequence alignment through sequence weighting, position-specific gap penalties and weight matrix choice. Nucleic Acids Res 22: 4673-4680, 1994

27. Mak KM, Ren C, Ponomarenko A, Cao Q and Lieber CS: Adipose differentiation-related protein is a reliable lipid droplet marker in alcoholic fatty liver of rats. Alcohol Clin Exp Res 32: 683-689, 2008.

28. Jensen-Seaman MI, Furey TS, Payseur BA, et al: Comparative recombination rates in the rat, mouse, and human genomes. Genome Res 14: 528-538, 2004.

29. Han L, Su B, Li WH and Zhao Z: CpG island density and its correlations with genomic features in mammalian genomes. Genome Biol 9: R79, 2008.

30. Nachman MW and Churchill GA: Heterogeneity in rates of recombination across the mouse genome. Genetics 142: 537-548, 1996.

31. Broman KW, Murray JC, Sheffield VC, White RL and Weber JL: Comprehensive human genetic maps: individual and sexspecific variation in recombination. Am J Hum Genet 63: 861$869,1998$.

32. Yu A, Zhao C, Fan Y, et al: Comparison of human genetic and sequence-based physical maps. Nature 409: 951-953, 2001.

33. Kong A, Gudbjartsson DF, Sainz J, et al: A high-resolution recombination map of the human genome. Nat Genet 31: 241-247, 2002.

34. Gomez-Lechon MJ, Donato MT, Martinez-Romero A, Jimenez N, Castell JV and O'Connor JE: A human hepatocellular in vitro model to investigate steatosis. Chem Biol Interact 165: 106-116, 2007.

35. Ricchi M, Odoardi MR, Carulli L, et al: Differential effect of oleic and palmitic acid on lipid accumulation and apoptosis in cultured hepatocytes. J Gastroenterol Hepatol 24: 830-840, 2009.

36. Boelsterli UA and Bedoucha M: Toxicological consequences of altered peroxisome proliferator-activated receptor gamma (PPARgamma) expression in the liver: insights from models of obesity and type 2 diabetes. Biochem Pharmacol 63: 1-10, 2002.

37. Inoue $\mathrm{M}$, Ohtake $\mathrm{T}$, Motomura $\mathrm{W}$, et al: Increased expression of PPARgamma in high fat diet-induced liver steatosis in mice. Biochem Biophys Res Commun 336: 215-222, 2005.

38. Wolins NE, Brasaemle DL and Bickel PE: A proposed model of fat packaging by exchangeable lipid droplet proteins. FEBS Lett 580: 5484-5491, 2006. 